\title{
ON STABLY EXTENDED PROJECTIVE MODULES OVER POLYNOMIAL RINGS
}

\author{
MOSHE ROITMAN
}

\begin{abstract}
We prove here that if $A$ is a commutative noetherian ring of Krull dimension $d$ and of finite characteristic prime to $d$ !, then stably extended projective $A\left[X_{1}, \ldots, X_{n}\right]$-modules of rank $\geq d / 2+1$ are extended from $A$.
\end{abstract}

We denote by $A$ a commutative ring with unit. $U_{r}(A)$ is the set of unimodular rows of length $r$ over $A$. As in $\left[3, \S 5\right.$, p. 34], given $u, v$ in $U_{r}(A)$ and a subgroup $G$ of $G L_{r}(A)$, we write $u \sim_{G} v$ if there exists $g$ in $G$ such that $v=u g$. We abbreviate the notations $u \sim_{G L_{r}(A)} v$ to $u \sim v$ and $u \sim_{E_{r}(A)} v$ to $u \sim_{E} v$. For $u, v$ in $U_{r}(A)$ we denote by $u \leftrightarrow G L_{r}(A) v$ or simply $u \leftrightarrow v$ the property: $u \sim(1,0, \ldots, 0)$ if and only if $v \sim(1,0, \ldots, 0)$.

If $\varphi: A \rightarrow B$ is a canonical ring homomorphism (such as $A \rightarrow A_{S}, A \rightarrow A / I$, where $S$ is a multiplicative subset and $I$ is an ideal of $A$ ) and $a \in A$, we denote $\varphi(a)=\bar{a}$.

If $f(X)$ is a polynomial in $A[X]$, we denote its leading coefficient by $l(f)$. As usual $A(X)$ denotes the localization of $A[X]$ at the set of monic polynomials. If $S$ is a multiplicative subset of $A$ and $f(X) \in A[X]$, we say that $f(X)$ is unitary in $A_{S}[X]$ if $\overline{f(X)}$ is unitary in $A_{S}[X]$, that is, $l(f)=u s$ for some $s \in S$ and $u$ invertible in $A$.

We recall that a finitely generated projective module $P$ over $R=A\left[X_{1}, \cdots, X_{n}\right]$ is called stably extended from $A$ if there exists a finitely generated $R$-projective module $Q$ extended from $A$ such that $P \oplus Q$ is extended from $A$ or, equivalently, if there exists $m \geq 0$ such that $P \oplus R^{m}$ is extended from $A$.

LeMma 1 (CF. [12, CoROllaRY 2]). Let $\left(x_{0}, \ldots, x_{r}\right) \in U_{r+1}(A), r \geq 2$, and let $t$ be an element of $A$ which is invertible $\bmod \left(A x_{0}+\cdots+A x_{r-2}\right)$. Then $\left(x_{0}, \ldots, x_{r}\right) \sim_{E}\left(x_{0}, \ldots, t^{2} x_{r}\right)$.

PROOF. Let $\sum_{i=0}^{r} x_{i} y_{i}=1$. Then by $[8$, Lemma 1$]$ we have

$$
\left(x_{0}, \ldots, x_{r-2}, x_{r-1}, x_{r}\right) \sim_{E}\left(x_{0}, \ldots, x_{r-2}, y_{r-1}, y_{r}\right) \sim_{E}\left(x_{0}, \ldots, x_{r-2}, t x_{r-1}, t x_{r}\right) .
$$

Let $t t^{\prime} \equiv 1 \bmod \left(A x_{0}+\cdots+A x_{r-2}\right)$. By Whitehead's lemma we have

$$
\begin{aligned}
\left(x_{0}, \ldots, x_{r-2}, t x_{r-1}, t x_{r}\right) & \sim_{E}\left(x_{0}, \ldots, x_{r-2}, t^{\prime} t x_{r-1}, t^{2} x_{r}\right) \\
& \sim_{E}\left(x_{0}, \ldots, x_{r-2}, x_{r-1}, t^{2} x_{r}\right) .
\end{aligned}
$$

LEMMA 2 (CF. E.G., [1, THÉORÈME 1]). Let $S$ be a multiplicative subset of $A$, such that $A_{S}$ is noetherian of finite Krull dimension d. Let $\left(\bar{a}_{0}, \ldots, \bar{a}_{r}\right) \in$

Received by the editors December 3, 1984 and, in revised form, May 6, 1985.

1980 Mathematics Subject Classification. Primary 13C10, 13 F20. 
$U_{r+1}\left(A_{S}\right), r>d$. Then there exist $b_{i} \in A(1 \leq i \leq r)$ and $s \in S$ such that $s \in A\left(a_{1}+b_{1} a_{0}\right)+\cdots+A\left(a_{r}+b_{r} a_{0}\right)$.

PROOF. Similar to that of $[1$, Théorème $1, \S 3]$. We have to choose elements in $A$ (as $a_{i}^{\prime \prime}$ in $[\mathbf{1}, \S 3$, Lemma 2]) in order to avoid certain prime ideals in $A$ which come from prime ideals in $A_{S}$. Finally we obtain $A_{S}\left(\overline{a_{1}+b_{1} a_{0}}\right)+\cdots+A_{S}\left(\overline{a_{r}+b_{r} a_{0}}\right)=$ $A_{S}$.

LEMMA 3. Let $f(X)$ be a polynomial in $R=A[X]$ of degree $n>0$, such that $f(0)$ is invertible in $A$. Then for any $g(X) \in A[X]$ and natural $k \geq \operatorname{deg} g(X)-$ $\operatorname{deg} f(X)+1$ there exists $h_{k}(X) \in A[X]$ of degree $<n$ such that $g(X)=X^{k} h_{k}(X)$ $\bmod (R f(X))$.

ProOF. Let $f(X)=a_{0}+\cdots+a_{n} X^{n}, g(X)=c_{0}+\cdots+c_{m} X^{m}$. Let $g(X)-$ $c_{0} a_{0}^{-1} f(X)=X h_{1}(X)$. Then $g(X) \equiv X h_{1}(X) \bmod (R f(X))$ and $\operatorname{deg} h_{1}(X)<$ $\max (m, n)$. Similarly we obtain $h_{2}(X)$ such that $h_{1}(X) \equiv X h_{2}(X) \bmod (R f(X))$, $g(X)=X^{2} h_{2}(X) \bmod (R f(X)), \operatorname{deg} h_{2}(X)<\max (m-1, n)$, etc. In this way the lemma easily follows.

LEMMA 4. Let $\left(x_{0}, \ldots, x_{r}\right) \in U_{r+1}(A)$ and $k \equiv 1 \bmod (r !)$. Then for any $0 \leq i \leq r$ we have $\left(x_{0}, \ldots, x_{i}, \ldots, x_{r}\right) \leftrightarrow\left(x_{0}, \ldots, x_{i}^{k}, \ldots, x_{r}\right)$.

PROOF. Let $i=0$. Any unimodular row which contains a $(k-1)$-power of an element in $A$ is completable to an invertible matrix by [9, Theorem 2]. It follows by [9, Corollary 3.3], that if $\left(x_{0}, \ldots, x_{r}\right) \sim(1,0, \ldots, 0)$, then $\left(x_{0}^{k}, \ldots, x_{r}\right) \sim$ $(1,0, \ldots, 0)$. On the other hand if $\left(x_{0}^{k}, \ldots, x_{r}\right) \sim(1,0, \ldots, 0)$, then let $x_{0} x_{0}^{\prime} \equiv$ $1 \bmod \left(A x_{1}+\cdots+A x_{r}\right)$ and so,

$$
\left(x_{0}, \ldots, x_{r}\right) \sim_{E}\left(x_{0}^{k} x_{0}^{\prime k-1}, \ldots, x_{r}\right) \sim(1,0, \ldots, 0)
$$

by $[\mathbf{9}$, Theorem 2] and [9, Corollary 3.3].

THEOREM 5 (CF. [1, THÉORÈME 3]). Let $A$ be a commutative noetherian ring of finite Krull dimension $d$, let $r \geq d / 2+1$ and assume that $A$ is of finite characteristic prime to $r$ !. Let $P$ be a finitely generated projective module of rank $r$ over $R=A\left[X_{1}, \ldots, X_{n}\right]$ such that $P \oplus R$ is extended from $A$. Then $P$ is extended from $A$.

Proof. By Quillen's Patching Theorem [6, Theorem $1^{\prime}$ or 3, Chapter $\left.5, \S 1\right]$ we may assume $A$ to be local, so $\operatorname{char} A$ is a power of a prime $p$. Let $P_{0}$ be the $A$-module $P /\left(X_{1} P+\cdots+X_{n} P\right)$. We have to show $P \cong P_{0} \otimes_{R} R$. As $p$ is in the nilradical of $R$, it is enough to show $P / p P \cong\left(P_{0} \otimes_{R} R\right) / p\left(P_{0} \otimes_{R} R\right)$ (see e.g. [3, Chapter 1, Corollary 1.6]). We have

$$
\frac{P_{0} \otimes_{R} R}{p\left(P_{0} \otimes_{R} R\right)} \cong \frac{P_{0}}{p P_{0}} \otimes_{A / p A} \frac{R}{p R} \quad \text { over } \frac{R}{p R}=\frac{A}{p A}\left[X_{1}, \ldots, X_{n}\right],
$$

which means that the $R / p R$-module $\left(P_{0} \otimes_{R} R\right) / p\left(P_{0} \otimes_{R} R\right)$ is extended from the $A / p A$-module $P_{0} / p P_{0}$. Therefore we have to show that the $R / p R$-module $P / p P$ is extended from $A / p A$. Replacing $A$ by $A / p A$, we assume char $A=p$. By the Quillen induction (see $[6$ or 3 , Chapter $5, \S 3]$ ) we reduce to the case $n=1, R=A[X]$ :

Let $n>1$ and let $S$ be the set of monic polynomials in $A\left[X_{1}\right], A\left(X_{1}\right)=A\left[X_{1}\right]_{s}$. Then $\operatorname{dim} A\left(X_{1}\right)=\operatorname{dim} A[3$, Chapter 4, Proposition 1.2] and so by induction the 
$A\left(X_{1}\right)\left[X_{2}, \ldots, X_{n}\right]$-module $P_{s}$ is extended from $A\left(X_{1}\right)$. By Horrocks' theorem (see e.g. [3, Chapter 4]), $P$ is extended from $A$.

We have to prove that $G L_{r+1}(R)$ acts transitively on $U_{r+1}(R)$. Let us call admissible transformations of a row $u \in U_{r+1}(R)$ elementary transformations and also transformations of the type

$$
\left(x_{0}, \ldots, x_{i}, \ldots, x_{r}\right) \rightarrow\left(x_{0}, \ldots, x_{i}^{k}, \ldots, x_{r}\right), \quad \text { where } k \equiv 1 \bmod (r !) .
$$

By Lemma 4 it is enough to prove the following

Claim. If $u(X)=\left(f_{0}(X), \ldots, f_{r+1}(X)\right) \in U_{r+1}(R), r \geq 2$, then $u$ can be transformed to $(1,0, \ldots, 0)$ using admissible transformations.

We prove the claim by induction on the number $N$ of nonzero coefficients of the polynomials $f_{0}(X), \ldots, f_{r}(X)$, starting with $N=1$. Let $N>1$. We may assume $\operatorname{deg} f_{0}>0$. Let $l\left(f_{0}\right)=a$. If $a$ is invertible, then $u \sim_{E}(1,0, \ldots, 0)$ (see, e.g., [2, Chapter III, Corollary 1.4]). In our case the proof is much simpler: As $f_{0}(X)$ is unitary and $A$ is local, there exist just a finite number of maximal ideals in $R$ which contain $f_{0}$, so there exists $g$ in $R$ which does not belong to any such ideal and $f_{1} \equiv g \bmod \left(R f_{2}+\cdots+R f_{r}\right)$. As $R f_{0}+R g=R$, we conclude $u \sim_{E}(1,0, \ldots, 0)$. We assume now that $a$ is not invertible in $A$. By the inductive assumption with respect to the ring $\bar{A}=A / a A$ and the row $\bar{u}(X)$, we can obtain from $u(X)$ a row $v(X) \equiv(1,0, \ldots, 0) \bmod (R a)$ using admissible transformations over $R$. We can perform such transformations so that at every stage the row contains a polynomial which is unitary in $R_{a}$. Indeed, if we have to perform, e.g., the elementary transformation

$$
\left(g_{0}, \ldots, g_{r}\right) \stackrel{T}{\rightarrow}\left(g_{0}, g_{1}+h g_{0}, \ldots, g_{r}\right)
$$

and $g_{1}$ is unitary in $R_{a}$, then we replace $T$ by the following two transformations:

$$
\begin{aligned}
\left(g_{0}, g_{1}, \ldots, g_{r}\right) & \rightarrow\left(g_{0}+a X^{m} g_{1}, g_{1}, \ldots, g_{r}\right) \\
& \rightarrow\left(g_{0}+a X^{m} g_{1}, g_{1}+h\left(g_{0}+a X^{m} g_{1}\right), \ldots, g_{r}\right),
\end{aligned}
$$

where $m>\operatorname{deg} g_{0}$. We assume now $\left(f_{0}, \ldots, f_{r}\right) \equiv(1,0, \ldots, 0) \bmod (R a)$ and $f_{i}$ is unitary in $R_{a}$. If $i>0$, then replace $f_{0}$ by $f_{0}+a X^{m} f_{i}$, where $m>\operatorname{deg} f_{0}$; so we assume $f_{0}$ is unitary in $R_{a}$ and also $\operatorname{deg} f_{0}>0$. By Lemma 3 we assume now $f_{i}=X^{2 k} g_{i}$, where $\operatorname{deg} g_{i}<\operatorname{deg} f_{i}$ for $1 \leq i \leq r$. By Lemma 1 we assume $\operatorname{deg} f_{i}<\operatorname{deg} f_{0}$ for $1 \leq i \leq r$.

Let $\operatorname{deg} f_{0}=m_{0}$. If $m_{0}=1$, then $f_{i} \in A$ for $1 \leq i \leq r$. Therefore for sufficiently big $q$ we have $\left(f_{0}(X)-f_{0}(0)\right)^{q} \in R f_{1}+\cdots+R f_{r}$. Choose such $q$ of the form $p^{n}$ and $q \equiv 1 \bmod (r !)$. Then we perform the admissible transformation $\left(f_{0}, \ldots, f_{r}\right) \rightarrow\left(f_{0}^{q}, \ldots, f_{r}\right)$. As char $A=p$, we have $f_{0}^{q}=f_{0}(0)+\left(f_{0}(X)-f_{0}(0)\right)^{q}$, so $\left(f_{0}^{q}, \ldots, f_{r}\right) \sim_{E_{n}(R)}(1,0, \ldots, 0)$.

Assume now $m_{0} \geq 2$. We use an argument similar to that in the proof of $[1, \S 4$, Theorem $3^{\prime}$. Let $\left(c_{1}, \ldots, c_{m_{0}(r-1)}\right)$ be the coefficients of $1, X, \ldots, X^{m_{0}-1}$ in the polynomials $f_{2}(X), \ldots, f_{r}(X)$. By [3, Chapter III, Lemma 1.1], the ideal generated in $A_{a}$ by $A_{a} \cap\left(R_{a} \bar{f}_{0}+R_{a} \bar{f}_{1}\right)$ and the coefficients of $\bar{f}_{i}(2 \leq i \leq r)$ is $A_{a}$. As $m_{0}(r-1) \geq 2 \cdot d / 2=d>\operatorname{dim} R_{a}$, by Lemma 2 there exists $\left(c_{1}^{\prime}, \ldots, c_{m_{0}(r-1)}\right) \equiv$ $\left(c_{1}, \ldots, c_{m_{0}(r-1)}\right) \bmod \left(\left(R f_{0}+R f_{1}\right) \cap A\right)$, such that $A_{a} \bar{c}_{1}^{\prime}+\cdots+A_{a} \bar{c}_{m_{0}(r-1)}^{\prime}=A_{a}$. Assume that we have already $A_{a} \bar{c}_{1}+\cdots+A_{a} \bar{c}_{m_{0}(r-1)}=A_{a}$. By $[\mathbf{1}, \S 4$, Lemma 1(b)], the ideal $A f_{0}+A f_{2}+\cdots+A f_{r}$ contains a polynomial $h(X)$ of degree $m_{0}-1$ 
which is unitary in $R_{a}$. Let $l(h)=u a^{k}$, where $u$ is invertible in $A$. Using Lemma 1 , we achieve by elementary transformations

$$
\begin{aligned}
\left(f_{0}, f_{1}, \ldots, f_{r}\right) & \rightarrow\left(f_{0}, a^{2 k} f_{1}, \ldots, f_{r}\right) \\
& \rightarrow\left(f_{0}, a^{2 k} f_{1}+\left(1-a^{k} u^{-1} l\left(f_{1}\right) h, \ldots, f_{r}\right)\right) .
\end{aligned}
$$

Now, $a^{2 k} f_{1}+\left(1-a^{k} u^{-1} l\left(f_{1}\right)\right) h$ is unitary in $R_{a}$, so assume $f_{1}$ is unitary in $R_{a}$, $\operatorname{deg} f_{1}=m_{1}<\operatorname{deg} f_{0}$. By Lemma 1 we assume also $\operatorname{deg} f_{i}<m_{1}$ for $2 \leq i \leq r$. Repeating the argument above we lower the degree of $f_{1}$ and obtain finally a row of the form $\left(f_{0}, f_{1}, \ldots, f_{r}\right) \equiv(1,0, \ldots, 0) \bmod (R a)$ with $f_{0}$ unitary in $R_{a}, m_{0}=$ $\operatorname{deg} f_{0}>\operatorname{deg} f_{1}=1$ and $f_{i} \in A$ for $2 \leq i \leq r$. Let $l\left(f_{1}\right)=u a^{k}$, where $u$ is invertible in $A, f_{0}(X)=1-a g(X)$. We have by Lemma 3

$$
f_{2} \equiv f_{2}+1-a^{k m_{0}} g^{k m_{0}} \equiv f_{2}+1-a^{k m_{0}} X^{q} h(X) \bmod \left(R f_{0}\right)
$$

for some $q$ of the form $p^{n}, q \equiv 1 \bmod (r !)$ and $h(X)$ of degree $<m_{0}$. As $\operatorname{deg} h(X)<$ $m_{0}$, we have $a^{k m_{0}} h(X) \equiv b \bmod \left(R f_{1}\right)$ for some $b \in A$, so we can obtain $f_{2}(X)$ of the form $f_{2}(X)=u+c X^{q}$ with $c \in A$, $u$ invertible in $A$. By admissible transformations $\left(f_{0}, f_{1}, \ldots, f_{r}\right) \rightarrow\left(f_{0}^{q}, f_{1}^{q}, \ldots, f_{r}\right)$ we obtain $f_{0}^{q}, f_{1}^{q} \in A\left[X^{q}\right]$. By Lemma 3 we obtain a row of the type $\left(c_{0}, c_{1}, f_{2}, c_{3}, \ldots, c_{r}\right)$, where $c_{i} \in A$, so by the argument in the case $m_{0}=1$ we finish the proof.

Using [11, Theorem 1.1] we obtain

COROLLARY 6. If $A$ is a noetherian ring of dimension d and of characteristic prime to $d$ !, then projective stably extended $A\left[X_{1}, \ldots, X_{n}\right]$-modules of rank $\geq d / 2+1$ are extended from $A$.

COROLlaRY 7. If $A$ is a noetherian ring of dimension 2 and of finite odd characteristic, then projective stably extended $A\left[X_{1}, \ldots, X_{n}\right]$-modules are extended from $A$ (that is, $A\left[X_{1}, \ldots, X_{n}\right]$ is a Hermite ring).

Finally, we obtain the following particular case of the Bass-Quillen conjecture:

COROLLARY 8. If $A$ is a noetherian regular ring of dimension $d$ and of characteristic prime to $d$ !, then finitely generated projective $A\left[X_{1}, \ldots, X_{n}\right]$-modules of rank $\geq d / 2+1$ are extended from $A$.

Without the assumption on the characteristic, Corollary 6 would strengthen Theorem 1.1 in [11] restricted to polynomial rings (see also [10, Theorem 7.2]) and Corollaries 7 and 8 would generalize the Murthy-Horrocks Theorem (see, e.g., [3, Chapter V, Theorem 3.3]).

It can be shown using [10, Theorem 7.2] and the arguments above that if $A$ is noetherian of dimension $d$ and $u \in U_{d+1}(A[X])$, then there exist $u_{1}, u_{2}$ in $U_{d+1}(A[X])$ such that $u \sim_{E} u_{1} \sim_{E} u_{2}, u_{1}$ is of the form $\left(1+a X^{n}, b+a X, c_{2}, \ldots, c_{r}\right)$ with $a, b, c_{2}, \ldots, c_{r}$ in $A$ and $u_{2}$ is of the form $\left(1+a X^{n}, 1+b X^{m}, c_{2}, \ldots, c_{r}\right)$ with $a, b, c_{2}, \ldots, c_{r}$ in $A$.

Finally we mention some related results. If we assume $A$ is regular, then projective $A\left[X_{1}, \ldots, X_{n}\right]$-modules are extended under certain additional assumptions not necessarily related to Krull dimension; e.g., by Lindel's theorem [4] this holds if $A$ is a regular algebra of essentially finite type over a field (see also [5]). For a survey of further results apart from those in $[\mathbf{3}]$ see $[\mathbf{1 3}]$. For more recent results see Suslin's work in Trudy Mat. Inst. Steklov. 168 (1984) (English translation to appear in Proc. Steklov Inst. Math.). See also [7]. 


\section{REFERENCES}

1. H. Bass, Libération des modules projectifs sur certains anneaux de polynômes, Sém. Bourbaki 1973/74, exp. 448, Lecture Notes in Math., vol. 431, Springer-Verlag, Berlin and New York, 1975, pp. 228-254.

2. S. K. Gupta and M. P. Murthy, Suslin's work on linear groups over polynomial rings and Serre problem, 151 Lecture Notes, No. 8, The Macmillan Company of India Limited, Delhi.

3. T. Y. Lam, Serre's conjecture, Lecture Notes in Math., vol. 635, Springer, 1978.

4. H. Lindel, Projective Moduln über Polynomring $A\left[T_{1}, \ldots, T_{m}\right]$ mit einem regulären Grundring A, Manuscripta Math. 23 (1978), 143-154.

5. __ On the Bass-Quillen conjecture concerning projective modules over polynomial rings, Invent. Math. 65 (1981), 319-323.

6. D. Quillen, Projective modules over polynomial rings, Invent. Math. 36 (1976), 167-171.

7. R. A. Rao, On projective $R_{f_{1} \cdots f_{t}}$-modules, Amer. J. Math. 107 (1985), 387-406.

8. M. Roitman, On unimodular rows, Proc. Amer. Math. Soc. 95 (1985), 184-188.

9. A. A. Suslin, On stably free modules, Mat. Sb. 102 (144) (1977), 537-550 (= Math. USSRSb. 31 (1977), 479-491).

10. SSSR Ser. Mat. 41 (1977), 235-252 (= Math. USSR Izv. 11 (1977), 221-238).

11. R. G. Swan, Projective modules over Laurent polynomial rings, Trans. Amer. Math. Soc. 237 (1978), 111-120.

12. L. N. Vaserstein, Operations on orbits of unimodular vectors, J. Algebra (to appear).

13. Ton Vorst, $A$ survey on the $K$-theory of polynomial extensions, Algebraic $K$-Theory, Number Theory, Geometry and Analysis (Proc. Internat. Conf., Bielefeld, Germany, 1982), Lecture Notes in Math., vol. 1046, Springer, 1984, pp. 422-441.

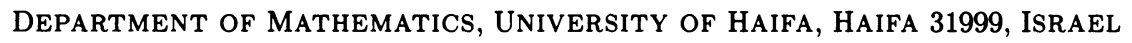

Current address: Department of Mathematics and Statistics, Queen's University, Kingston, Ontario, Canada K7L 3N6 